\title{
Magnetometer Calibration Algorithm Based on Analytic Geometry Transform
}

\author{
Yongjian Yang, Xiaolong $\mathrm{XiaO}^{1}$, Wu Liao \\ College of Computer Science and Technology, Jilin University, Changchun 130012, China \\ ${ }^{1}$ xiaoxl13@163.com
}

\begin{abstract}
Keywords: magnetometer calibration, tilted ellipse, analytic geometry, geometry transform Abstract: Based on the analysis of Hard- and Soft- Iron effects of magnetometer's errors, a tilted ellipse model for two-dimensional plane was formulated. According to the analytic geometry theory, a magnetometer calibration algorithm was proposed to calibrate the tilted ellipse into a standard circle by translation, rotation and scaling three steps, achieving the aim of magnetometer calibration. The experimental results show that the proposed algorithm calibrated the tilted ellipse approximately to a standard circle which had eliminated the combined Hard- and Soft- Iron effects successfully.
\end{abstract}

\section{Introduction}

Magnetometers measure the strength of magnetic fields and widely applied in science, engineering[1]. According to the World Magnetic Model[2], the strength of geomagnetic field in a place is constant, so magnetometers are often used to measure it to determine the geographic orientation. But errors occur as magnetometer readings are also subject to magnetic distortions and nonideal manufacture techniques such as zero-offset, hard iron, soft iron, scaling and non-orthogonality, the first two can be grouped as Hard-Iron offset, remaining as Soft-Iron effect[3]. Then calibration algorithm is needed to eliminate them, [4] used a compass swinging algorithm for two-dimensional heading determination system, [5] used recursive least square algorithm for three-dimensional magnetic deviation compensation, [6] used ellipse matching error compensation algorithm for two-dimensional magnetic compass; For simplicity, this paper first proposed a two-dimensional algorithm based on analytic geometry transform. Ideally, The readings $\left(H_{\mathrm{mx}}[\mathrm{i}]\right.$, $\left.H_{\text {my }}[\mathrm{i}]\right)$ from a magnetometer when rotating in the horizontal plane is a standard circle. Given the errors, the circle is transformed into a tilted ellipse. The proposed algorithm is based on this geometry model, transforming the tilted ellipse into the circle by translation, rotation, scaling to achieve magnetometer calibration. Experimental results show that the algorithm performed well to calibrate magnetometers in reality.

This paper is organized as follows. In the next section, magnetometer errors are analyzed, a calibration model is formulated. The detailed algorithm is presented in the third section. Experimental results obtained with a low-cost magnetometer are presented and discussed in the fourth section. The last section draws concluding remarks and comments on future work.

\section{Magnetometer errors and calibration model}

In this section, magnetometer errors are analyzed, a calibration model is formulated. Magnetometer errors

1) Hard-Iron offset

The zero-offset is a vector $\boldsymbol{V}_{\text {zero, }}$, the permanently magnetized ferromagnetic components in the vicinity of a magnetometer is a vector $\boldsymbol{V}_{\mathrm{h}}$. Both $\boldsymbol{V}_{\text {zero }}$ and $\boldsymbol{V}_{\mathrm{h}}$ are time invariant, and simply add to the readings which can be grouped as Hard-Iron offset $\boldsymbol{V}$.

$$
\boldsymbol{V}=\boldsymbol{V}_{\text {zero }}+\boldsymbol{V}_{\mathrm{h}}
$$

2) Soft-Iron effect

A linear time-varying magnetic field generated by magnetically soft materials is soft iron 
distortion which can be modeled by a $2 \times 2$ matrix $\boldsymbol{W}_{\text {soft }}$. The sensitivities of each sensing axis are not exactly equal, a $2 \times 2$ diagonal gain matrix $\boldsymbol{W}_{\text {gain }}$ can be modeled. The last non-orthogonality error can be modeled by a $2 \times 2$ matrix $\boldsymbol{W}_{\text {no }}$ caused by non-orthogonal sensing axes installation. Soft iron, scaling and non-orthogonality can be grouped as Soft-Iron effect, as they together make a standard circle become a tilted ellipse. Then the Soft-Iron matrix is Eq. 2.

$$
W=W_{\text {no }} W_{\text {gain }} W_{\text {soft }}
$$

Magnetometer calibration model

Given the combined effects of Hard- and Soft- Iron, a model between magnetometer readings $\boldsymbol{H}_{\mathrm{m}}$ and the true local geomagnetic $\boldsymbol{H}_{\mathrm{r}}$ is formulated as Eq. 3.

$$
\boldsymbol{H}_{\mathrm{m}}=\boldsymbol{W H}_{\mathrm{r}}+\boldsymbol{V}
$$

Derived from Eq. 3,

$$
\begin{aligned}
& \boldsymbol{H}_{r}=\boldsymbol{W}^{-1}\left(\boldsymbol{H}_{m}-\boldsymbol{V}\right) \\
& \begin{aligned}
\left\|\boldsymbol{H}_{r}\right\|^{2} & =\boldsymbol{H}_{r}{ }^{T} \boldsymbol{H}_{r}=\left\{\boldsymbol{W}^{-1}\left(\boldsymbol{H}_{m}-\boldsymbol{V}\right)\right\}^{T} \boldsymbol{W}^{-1}\left(\boldsymbol{H}_{m}-\boldsymbol{V}\right) \\
& =\left(\boldsymbol{H}_{m}-\boldsymbol{V}\right)^{T}\left(\boldsymbol{W}^{-1}\right)^{T} \boldsymbol{W}^{-1}\left(\boldsymbol{H}_{m}-\boldsymbol{V}\right)
\end{aligned}
\end{aligned}
$$

As the true local geomagnetic $\boldsymbol{H}_{\mathrm{r}}$ is a constant vector, and the matrix $\boldsymbol{A}$ in Eq. 6 is symmetric,

$$
\boldsymbol{A}=\left(\boldsymbol{W}^{-1}\right)^{T} \boldsymbol{W}^{-1}
$$

The general matrix form expression defining the locus of a vector $\boldsymbol{Q}$ wandering on the curve of an ellipse with center at $\boldsymbol{Q}_{0}$ is (where $\boldsymbol{A}$ must be a symmetric matrix):

$$
\left(\boldsymbol{Q}-\boldsymbol{Q}_{0}\right)^{T} \boldsymbol{A}\left(\boldsymbol{Q}-\mathbf{Q}_{0}\right)=\text { const }
$$

As Eq. 5 and Eq. 7 are similar, the locus of magnetometer readings is an ellipse defined by Eq. 5 . The ellipse is centered at the Hard-Iron offset $\boldsymbol{V}$, and has a tilted shape determined by matrix $\boldsymbol{A}=$ $\left(\boldsymbol{W}^{-1}\right)^{\mathrm{T}} \boldsymbol{W}^{-1}$.

\section{Algorithm implementation details}

In this section, the details of proposed algorithm is presented.

According to the geometric theory, the max distance between two points on an ellipse curve is the length of the major-axis, the two points are the two end points of the major-axis, and the center of the two points is the very center of ellipse. Using the readings collected by the magnetometer and Eq. 8

$$
2 a=\max \left(\sqrt{\left(H_{m x}[i]-H_{m x}[j]\right)^{2}+\left(H_{m y}[i]-H_{m y}[j]\right)^{2}}\right)
$$

to find the two points $\left(H_{m x}\left[a_{1}\right], H_{m y}\left[a_{1}\right]\right),\left(H_{m x}\left[a_{2}\right], H_{m y}\left[a_{2}\right]\right)$.

Then the center of the ellipse or the Hard-Iron offset is: 


$$
\boldsymbol{V}_{0}=\left(\left(H_{m x}\left[a_{1}\right]+H_{m x}\left[a_{2}\right]\right) / 2,\left(H_{m y}\left[a_{1}\right]+H_{m y}\left[a_{2}\right]\right) / 2\right)^{T}
$$

Using $\boldsymbol{H}_{\mathrm{m}}-\boldsymbol{V}_{0}$ translation to move the ellipse to the origin, as is shown in Fig. 1.

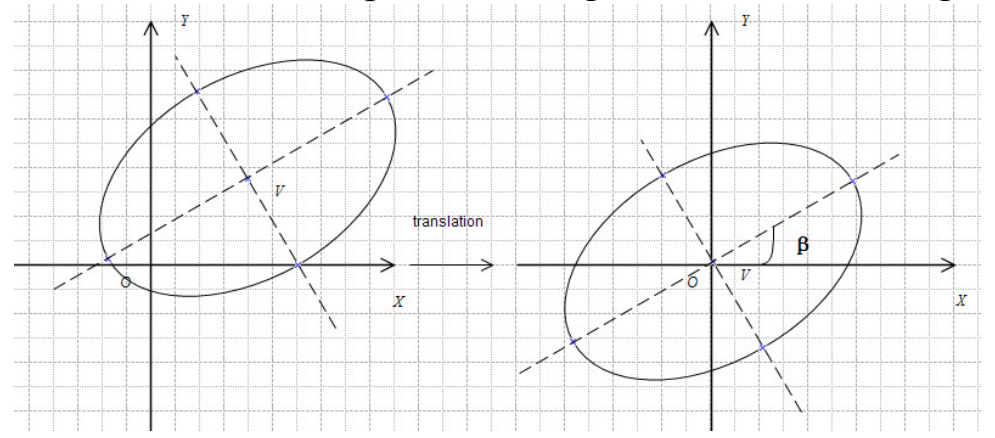

Fig. 1 Translation of Ellipse

The tilted or rotation angle $\beta$ of the ellipse can also be derived from Fig. 1,

$$
\begin{aligned}
& \sin \beta=\left|H_{m y}\left[a_{1}\right]-v_{21}\right| / a=k_{1} \\
& \cos \beta=\left|H_{m x}\left[a_{1}\right]-v_{11}\right| / a=k_{2}
\end{aligned}
$$

where $\boldsymbol{v}_{11}$ and $\boldsymbol{v}_{21}$ are the elements of $\boldsymbol{V}_{0}, a$ is the length of semi-major axis.

Based on the rotation theory of two-dimensional space[7], rotating a tilted ellipse back to a standard ellipse need to premultiply a rotation matrix defined in Eq. 11.

$$
\boldsymbol{R}(-\beta)=\left[\begin{array}{cc}
\cos \beta & \sin \beta \\
-\sin \beta & \cos \beta
\end{array}\right]=\left[\begin{array}{cc}
k_{2} & k_{1} \\
-k_{1} & k_{2}
\end{array}\right]
$$

By rotation of $\boldsymbol{H}_{e}=\boldsymbol{R}(-\beta) \cdot\left(\boldsymbol{H}_{m}-\boldsymbol{V}_{0}\right)$ to obtain a standard ellipse.

Given the properties of standard ellipse, the scale factors of $\boldsymbol{X}$ and $\boldsymbol{Y}$ axis are:

$$
\begin{aligned}
& X_{\text {scale }}=\max \left(1,\left(H_{e y \max }-H_{e y \min }\right) /\left(H_{e x \max }-H_{e x \min }\right)\right) \\
& Y_{\text {scale }}=\max \left(1,\left(H_{e x \max }-H_{e x \min }\right) /\left(H_{e y \max }-H_{e y \min }\right)\right)
\end{aligned}
$$

where $H_{\text {eymax }}$ is the max element of vector $\boldsymbol{H}_{\mathrm{e}}$, so it is with the other.

$$
\boldsymbol{W}_{\text {scale }}=\left[\begin{array}{cc}
X_{\text {scale }} & 0 \\
0 & Y_{\text {scale }}
\end{array}\right]
$$

Finally

$$
\boldsymbol{H}_{r}=\boldsymbol{W}_{\text {scale }} \cdot \boldsymbol{R}(-\beta) \cdot\left(\boldsymbol{H}_{m}-\boldsymbol{V}_{0}\right)
$$

The proposed algorithm can be summarized as follows:

1) Using Eq. 8, 9 to find the Hard-Iron offset $\boldsymbol{V}_{0}$, then use translation to obtain 
$\boldsymbol{H}_{\mathrm{m}}-\boldsymbol{V}_{0}$

2) Using Eq. 10, 11 to find the rotation matrix $\boldsymbol{R}(-\beta)$, then use rotation to obtain $\boldsymbol{H}_{e}=\boldsymbol{R}(-\beta) \cdot\left(\boldsymbol{H}_{m}-\boldsymbol{V}_{0}\right)$;

3) Using Eq. 12, 13 to find the scale factor, finally to obtain the calibrated value $\boldsymbol{H}_{r}=\boldsymbol{W}_{\text {scale }} \cdot \boldsymbol{R}(-\boldsymbol{\beta}) \cdot\left(\boldsymbol{H}_{m}-\boldsymbol{V}_{0}\right)$, and find the solution of the aforementioned calibration model compared with Eq. 4 :

$$
\left\{\begin{array}{c}
\boldsymbol{V}=\boldsymbol{V}_{0} \\
\boldsymbol{W}^{-1}=\boldsymbol{W}_{\text {scale }} R(-\beta)
\end{array}\right.
$$

\section{Experimental results}

In this section, the proposed algorithm is validated using experimental data from a low-cost magnetometer installed on a wireless sensor node.

A set of 2000 data points obtained from a AK8963 3-axis magnetometer(only the X and Y axes data in the horizontal plane were recorded) with a $0.15 \mu \mathrm{T} / \mathrm{LSB}$ sensitivity, sampled with a ATmega328 MCU(Micro Controller Unit), transmitted by a Xbee24 RF(Radio Frequency) module, at $100 \mathrm{~Hz}$. The hardware is shown in Fig. 2.

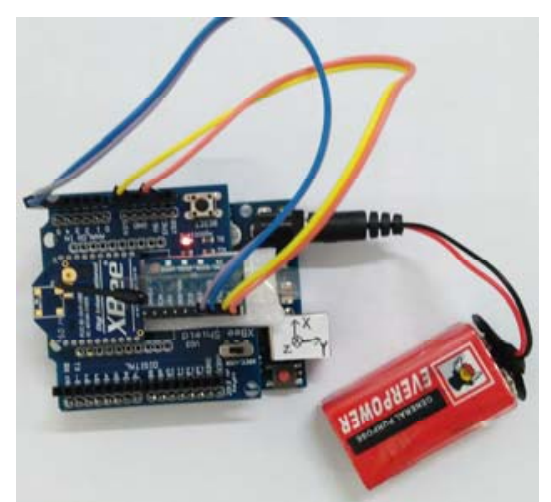

Fig. 2 Wireless Sensor Node

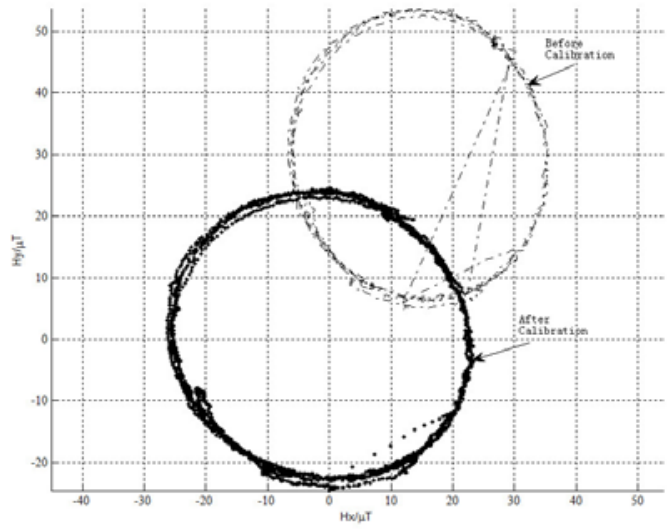

Fig. 3 Contrast through Calibration

By programming matlab codes to implement the proposed algorithm and running on the raw data set to obtain the solution:

$$
\left\{\begin{array}{c}
\boldsymbol{V}=\boldsymbol{V}_{0}=(15.7350,29.2050)^{T} \\
\boldsymbol{W}^{-1}=\boldsymbol{W}_{\text {scale }} \boldsymbol{R}(-\beta)=\left[\begin{array}{cc}
1.1432 & -0.1696 \\
0.1468 & 0.9892
\end{array}\right]
\end{array}\right.
$$

Using Eq. 4, 16 to calibrate the raw data is shown in Fig.3.

As is shown in Fig. 3, the calibrated data locus was approximate to a standard circle, which validates the proposed algorithm which had eliminated the combined Hard- and Soft- Iron effects successfully.

\section{Conclusions}

The Hard- and Soft- Iron effects of magnetometer errors were analyzed, the magnetometer calibration model was formulated, and it was shown that the magnetometer raw measurement data sets form a tilted ellipse. The solution of the experimental data set was derived and tested using matlab codes. The proposed magnetometer calibration algorithm based on analytic geometry transform was derived. Experimental results for low-cost magnetometer show that the proposed 
algorithm performed well by using translation, rotation, scaling to eliminate the Hard- and SoftIron effects errors for two-dimensional magnetometer readings. Future work will include the expansion of the proposed algorithm to the three dimensional case.

\section{Acknowledgements}

This work is supported by National Natural Science Foundation of China (No. 61272412).

\section{References}

[1] Information on https://en.wikipedia.org/w/index.php?title=Magnetometer

[2] Information on http://www.ngdc.noaa.gov/geomag/WMM/DoDWMM.shtml.

[3] Gebre-Egziabher, Demoz, Gabriel H. Elkaim, J. David Powell, and Bradford W. Parkinson 2006 Journal of Aerospace Engineering 19(2): 87-102.

[4] Nathaniel Bowditch. The American Practical Navigator. Defense Mapping Agency, Hydrographic/Topographic Center, Bethesda, Maryland, USA, 1995.

[5] Pengfei Guo, Chunhong Hua, Zhang Ren, Xinchun Ding. Magnetic deviation compensation using recursive least square for AHRS [J]. Journal of Chinese Inertial Technology. 2008(01). In Chinese.

[6] Yong Qin, Jie Zhao, Xiaoyu Wang. Digital magnetic compass based on ellipse matching error compensation algorithm[J]. Journal of Jilin University: Engineering and Technology Edition, 2009, 39(2): 489-493. In Chinese.

[7] Information on https://en.wikipedia.org/w/index.php?title=Rotation_matrix 